\title{
Distinguishing Collisions from Low Signal Strength in Static 802.11n Wireless LANs
}

\author{
†Mingu Cho, †Hakyung Jung, †Shinhaeng Oh, ‡Ted “Taekyoung” Kwon, `Yanghee Choi \\ School of Computer Science and Engineering \\ Seoul National University, Seoul, Korea \\ \{mgcho, hkjung, shoh\}@mmlab.snu.ac.kr ${ }^{\dagger},\left\{\right.$ tkkwon, yhchoi\}@snu.ac.kr ${ }^{\ddagger}$
}

\begin{abstract}
This paper proposes how to figure out whether frame losses are due to collisions or low signal strength. We observe subframe losses have distinct patterns through experiments, and devise a simple criterion which effectively distinguishes collisions from weak signals.
\end{abstract}

\section{INTRODUCTION}

In wireless network, frames can be lost due to either collisions or weak signals. When a frame transmission fails, stations can take different actions depending on the reasons for frame losses. In case of a loss due to weak signals, adaptation of data-rate can be performed. For a frame loss due to collisions, the contention window should be doubled to mitigate collisions. Therefore, it is crucial for 802.11 networks to differentiate collisions from weak signals.

In original IEEE 802.11 protocol, the only way to detect a frame loss at the sender side is the ACK frame from the receiver. Since an ACK frame is absent for either collisions or weak signals, it is difficult for the sender to accurately figure out the cause of the loss. To cope with this limitation, some previous studies seek to solve the problem with the aid of the physical layer. For example, they used the magnitude of signal strength of each symbol [2] or bit [4] to figure out the reason of a frame loss, and modified the 802.11 standard to convey the diagnosis to the sender. However, these approaches cannot be implemented upon the commodity hardware. Rate adaptation algorithms like [1] tried to detect collisions by using RTS probing, but they may fail in the presence of hidden terminals.

Through comprehensive measurements on pre- $802.11 \mathrm{n}$

Permission to make digital or hard copies of all or part of this work for personal or classroom use is granted without fee provided that copies are not made or distributed for profit or commercial advantage and that copies bear this notice and the full citation on the first page. To copy otherwise, to republish, to post on servers or to redistribute to lists, requires prior specific permission and/or a fee.

ACM CoNEXT Student Workshop, December 6, 2011, Tokyo, Japan. Copyright 2011 ACM 978-1-4503-1042-0/11/0012 ...\$10.00.

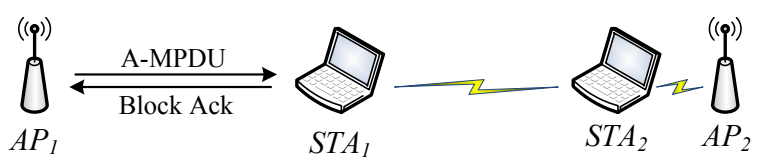

Figure 1: Experiment setup designed for inferring the reason of frame losses from $A P_{1}$ to $S T A_{1}$. Block ACK bitmaps are collected at $A P_{1}$.

hardware, we observe that the subframe loss patterns in case of collisions show burstiness whereas subframe losses due to weak signals occur independently. Based upon this observation, we propose a simple criterion which effectively separates collisions from weak signals after examining the loss pattern of a Block ACK bitmap. Since our approach only needs Block ACK bitmaps which are available at the sender in the current 802.11n standard, it can be easily employed by updating device drivers.

\section{COLLISION INFERENCE}

\section{Experiment Design.}

Figure 1 shows the experiment design to generate collisions between two Basic Service Sets (BSSs). One BSS is composed of $A P_{1}$ and $S T A_{1} . A P_{1}$ transmits A-MPDUs at a fixed data-rate (i.e., MCS 9) to $S T A_{1}$, which responds to Block ACK frames. We collected Block ACK bitmaps at $A P_{1}$ by modifying the Atk9k driver $^{1}$. In the first setting, the signal between $A P_{1}$ and $S T A_{1}$ is strong enough not to incur frame errors due to weak signals. The other BSS consisting of $A P_{2}$ and $S T A_{2}$ is located out of carrier sensing range of $A P_{1}$, but within transmission range of $S T A_{1}$. Thus, the traffic generated by $S T A_{2}$ acts as interference from hidden terminals. This ensures that no subframe errors at $S T A_{1}$ result from weak signals.

In the second setting, we attach an attenuator to $A P_{1}$, such that weak signals arrive at $S T A_{1}$, and we turn off

\footnotetext{
${ }^{1}$ We intentionally do not use sniffers for collecting bitmaps since it is known that the packet loss correlation is low even between closely located nodes [3]
} 


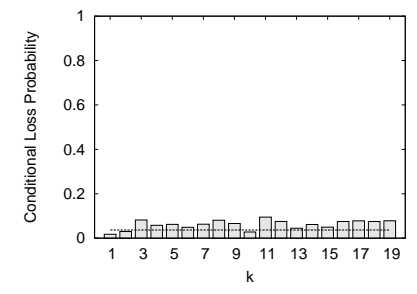

(a) $P\left(l_{i}\right)=0.04, I F=0$

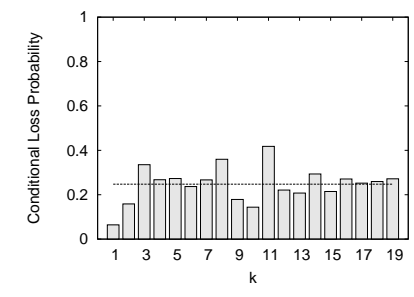

(b) $P\left(l_{i}\right)=0.25, I F=0$

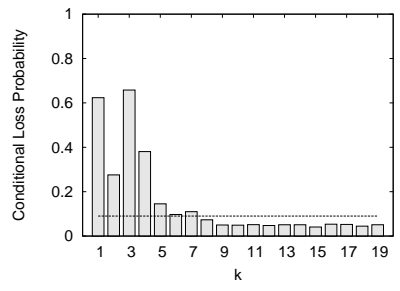

(c) $P\left(l_{i}\right)=0.09, I F=1 \mathrm{Mbps}$

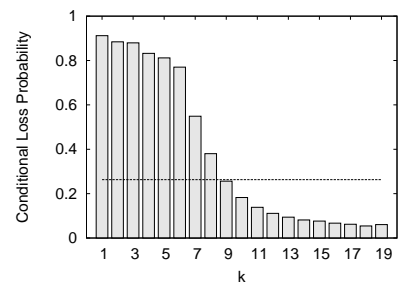

(d) $P\left(l_{i}\right)=0.26, I F=5 \mathrm{Mbps}$

Figure 2: Probability that subframe $i+k$ is lost, given that subframe $i$ was lost. Horizontal dashed lines indicate the overall loss probability $P\left(l_{i}\right)$, and $I F$ means the traffic load generated at the interferer, $S T A_{2}$.

$S T A_{2}$. We also verify that there is no other traffic using a separate sniffer.

\section{Empirical Analysis.}

Subframe losses due to collisions are observed to be bursty since the source of wireless interference normally generates traffic in a bursty manner. In order to quantify the loss burstiness, we adopted the concept of memorybased loss defined as $P\left(l_{i+k} \mid l_{i}\right)$, which is the probability of missing the $(i+k)^{t h}$ subframe given that the $i^{\text {th }}$ subframe is the first lost subframe within the bitmap. For small values of $k$, if the $P\left(l_{i+k} \mid l_{i}\right)$ is far greater than the overall loss probability $P\left(l_{i}\right)$, then the loss pattern is said to be bursty or memory-based.

Figures 2a and 2b show memory-based loss properties due to weak signals in low and high error environments, respectively. Both figures show no tendency towards memory-based loss. Loss probabilities across all the values of $k$ are similar with the overall loss probability, indicating that each loss occurs independently. This tendency is the same regardless of the overall loss probability. On the other hand, loss patterns due to collisions indicate strong tendency towards memory-based loss as shown in Figures 2c and 2d. For small values of $k, P\left(l_{i+k} \mid l_{i}\right)$ is far greater than the overall loss probability. In addition, the high value of $P\left(l_{i+k} \mid l_{i}\right)$ sustains for larger values of $k$ as the load of interference traffic is higher.

\section{Collision Inference.}

Based upon the above observations, we devise a simple criterion for inferring collisions. Given the overall loss probability ${ }^{2}$, we examine the bitmap of the target Block ACK frame. If the longest error burst within the bitmap is composed of $n$ consecutive zeros (i.e., subframe losses), we calculate the probability of such an event occurrence assuming the independent losses i.e., $P\left(l_{i}\right)^{n}$. If $P\left(l_{i}\right)^{n}<\epsilon$, we conclude that the losses were due to collisions since this rarely results from weak sig-

\footnotetext{
${ }^{2}$ In practice, the overall loss probability can be easily obtained by observing the previous A-MPDUs.
}

nals. After setting $\varepsilon=10^{-2}$, we evaluate the performance of our collision inference method using the trace data. We find that our method has an accuracy ranging $70-77 \%$ and a false positive rate ranging $0.4-2.9 \%$.

\section{FUTURE WORK}

We presented preliminary results on identifying the cause of frame losses (collision vs. weak signal) using the bitmap pattern conveyed within Block ACK frames. We will study the effect of subframe size, the number of aggregated subframes within an A-MPDU, and the appropriate value of $\epsilon$ depending on settings.

\section{ACKNOWLEDGMENTS}

This work was supported by NAP of Korea Research Council of Fundamental Science \& Technology.

\section{REFERENCES}

[1] J. Kim, S. Kim, S. Choi, and D. Qiao. Cara: Collision-aware rate adaptation for ieee 802.11 wlans. In INFOCOM 2006. 25th IEEE International Conference on Computer Communications. Proceedings, pages $1-11$, april 2006.

[2] S. Rayanchu, A. Mishra, D. Agrawal, S. Saha, and S. Banerjee. Diagnosing wireless packet losses in 802.11: Separating collision from weak signal. In INFOCOM 2008. The 27th Conference on Computer Communications. IEEE, pages $735-743$, april 2008.

[3] D. C. Salyers, A. D. Striegel, and C. Poellabauer. Wireless reliability: Rethinking 802.11 packet loss. A World of Wireless, Mobile and Multimedia Networks, International Symposium on, 0:1-4, 2008.

[4] M. Vutukuru, H. Balakrishnan, and K. Jamieson. Cross-layer wireless bit rate adaptation. In Proceedings of the ACM SIGCOMM 2009 conference on Data communication, SIGCOMM '09, pages 3-14, New York, NY, USA, 2009. ACM. 